\title{
La hibridación tecnológica Una aproximación al proceso comunicativo desde la perspectiva educativa
}

\author{
NANCY BALLESTAS \\ PABLO EMILIO RIVERA \\ Fecha de recepción: 30-06-2009 - Aprobación: 17-07-2009
}

\section{Resumen}

El siguiente artículo presenta un reflexión académica, producto del avance investigativo sobre la relación entre comunicación, tecnología y educación. En él se desarrolla una propuesta que hace evidente la importancia de comprender la manera en la que las mediaciones de carácter pedagógico y tecnológico se convierten en reguladores de los procesos de enseñanza-aprendizaje.

Así mismo, se pone en discusión el uso de plataformas educativas como estrategias y recursos de validación de las nuevas industrias culturales que encuentran en la exploración de los saberes un escenario para consolidar nuevas estructuras de mercado.

Este planteamiento conduce entonces a pensar lo social y su configuración. Su comprensión, hoy, implica dar cuenta de otras realidades de interacción social $y$, por ende, de otras formas de control social y de presencialidad de lo tecnológico como un componente vital para las relaciones y configuraciones de redes sociales. En este sentido, el escenario educativo es propicio para explorar esta configuración de lo social a partir del concepto "red" y, por tanto, permite revaluar sus propias mediaciones.

\section{Palabras clave}

Mediación, hibridación, modelo pedagógico.

\section{Abstract}

The following article presents an academic reflection, product of the research advance in the Communication/Technology/Education relation. This article develops a proposal that makes evident the importance of understanding the way the mediations of pedagogical and technological character become regulators of the education-learning processes.

It also discusses the implementation of educative platforms as strategies and validation resources of the new cultural industries that find in the exploration of knowledge a new scene to consolidate new market structures.

This approach leads to analyze the social aspect and its configuration since the understanding of that social denomination today involves the platforms to establish some other realities of social interaction and therefore some other forms of social control. It also takes into account the presence of the technological aspect as a vital component for the relations and the configurations of social networks. In this sense, the educative scene is favorable to explore this social configuration from the network concept; therefore, it allows revaluating these mediations to obtain the same network.

\section{Key words}

Mediation, hybridization, pedagogical model. 


\section{INTRODUCCIÓN}

El nuevo escenario educativo de la sociedad occidental, propio del siglo XX y XXI, incorpora la tecnología como elemento revolucionario de las formas de apropiación, consolidación y construcción del saber, así como una estrategia para acceder a experiencias académicas en procura del mejoramiento de la calidad de vida y el acceso a oportunidades de desarrollo personal y profesional.

Teniendo en cuenta la anterior premisa, la discusión se propone desde una perspectiva real; la constante transformación de las industrias culturales que se incorporan a escenarios tradicionalmente institucionalizados, definidos desde estructuras de valores que buscaban la uniformidad de comportamientos y vivencias para consolidar una sociedad ampliamente democrática y libre.

En este sentido, la lectura y la propuesta que se hace del concepto de industria cultural, definida y emergente en la Escuela de Frankfurt, se revalúa hoy, ya no como un síntoma de sin futuro, sino todo lo contrario, como un conjunto de oportunidades para que en la sociedad circulen todo tipo de bienes y servicios, que además cuentan hoy con un escenario mucho más abierto y mediático como la internet.

Este planteamiento desde la teoría crítica y la industria cultural nacida en este contexto propone preguntarse sobre la validez misma de la teoría y una discusión de fondo que implica legitimar esta forma de producción de bienes y servicios, ya no desde la perspectiva puramente ideológica, sino desde experiencias hoy profundamente marcadas por la estética del mercado de consumo, la satisfacción de necesidades reales y vitales, la utopía construida desde los mercados para abonar a la tendencia del mejoramiento de la calidad de vida o del ideal desarrollista al que le apuesta la modernidad en las proyecciones latinoamericanas.

La discusión parte de allí porque si bien existe una fuerte crítica a la Escuela de Frankfurt por su visión desalentadora del mundo y por la cosificación misma de la cultura, también es real que esta visión obedece a escenarios ampliamente definidos que el funcionalismo y estructuralismo norteamericanos rebaten a partir de una lectura absoluta de los procesos de comunicación.

En la reflexión que se adelanta, existen una serie de afirmaciones propuestas por varios autores como
Castells, Martín-Barbero, Rama, Adorno, Benjamin y otros. En estas, se hace pertinente tratar el tema de la hibridación tecnológica, que como concepto y como campo, aún no está propuesto de manera explícita, pero en ningún momento riñe con su concepción como industrial cultural: por el contrario se convierte en un camino viable para aproximarse al entendimiento de estas complejidades comunicativas y pedagógicas y valida la industria cultural como un mecanismo propio de las sociedades contemporáneas para consolidar un modelo económico y un discurso de la globalización, así como una posible respuesta desde la glocalización.

\section{NANCY BALLESTAS CARO}

Docente del Politécnico Grancolombiano, de la Facultad de Ciencias de la Comunicación, Departamento académico de Comunicación. Comunicadora social, egresada de la Universidad Los Libertadores. Maestría en Comunicación de la Universidad Católica de Lovaina, (Bélgica) y doctorada en Comunicación de la misma universidad.

nballestas@poligran.edu.co,caronancy@gmail.com

\section{PABLOEMILIO RIVERA}

Docente del Politécnico Grancolombiano, Departamento de Humanidades. Publicista, egresado de la Universidad de Bogotá Jorge Tadeo Lozano. Cuenta con una especialización en Comunicación Educativa de la Universidad Uniminuto.

pablobar@poligran.edu.co

\section{Desde las premisas básicas hacia una aproximación al concepto de hibridación tecnológica}

En el siguiente apartado, el texto aborda las premisas sobre las que se han ido construyendo las aproximaciones conceptuales y aplicativas de la hibridación tecnológica. En este sentido, esta manera de abordar la aproximación a una definición implica rastrear las posibilidades que se han ido conformando para comprender la dimensión de mediación comunicativa y mediación pedagógica.

Una primera aproximación que puede hacerse visible en el panorama actual de las propuestas académicas de educación superior es la del planteamiento sobre la relación entre bienes culturales y convergencias tecnológicas. A propósito de esta característica afirma Claudio Rama: 
Los desarrollos tecnológicos a través de internet y de los nuevos bienes culturales digitales, están planteando la confluencia entre la educación y la cultura y promoviendo en esta convergencia, tanto la virtualización de la educación como el desarrollo de las industrias culturales digitales con contenidos y dinámicas educativas de tipo interactivas que nos pudieran permitir hablar del nacimiento de las industrias educativas. (2007).

Esta afirmación da cuenta de una clara percepción sobre la imperiosa necesidad que existe en la sociedad actual de dar respuesta a dos escenarios que cada vez son más complementarios. El primer escenario, el de la educación, comprendida como un derecho y como el espacio propicio para la construcción de proyecto nación, proyecto académico y proyecto constructor de conocimiento. El segundo escenario, el del mercado. Este escenario visto como un espacio adverso y configurado desde las políticas de la globalización, de los grandes consorcios y desde las brechas que se reflejan fundamentalmente en la vida social. Este escenario vive una transformación porque, desde la perspectiva misma del mercado como circulación de bienes y servicios, está también abierto a que él sea asumido como un flujo de todo tipo de propuestas.

En este orden de ideas, ambos escenarios confluyen y encuentran un nicho propicio para adentrarse a discusiones de carácter social, en tanto que garantizar el derecho a la educación implica generar dinámicas de desarrollo, que de una u otra manera deben ser lideradas, financiadas y circularizadas con el fin de responder a la necesidad y la demanda educativa.

Crear esta industria educativa puede percibirse como una contradicción, pero en las dinámicas contemporáneas esta contradicción no existe y por el contrario cada vez más se valida esta posibilidad. Con ello también se busca generar un espacio de reconocimiento construido mediante un discurso que toca directamente la problemática social y la manera como la academia busca contribuir desde su criterio fundamental de responsabilidad social.

Este discurso de la responsabilidad social es también una categoría implícita en la propuesta de la hibridación tecnológica, en tanto que poner en evidencia el uso de la tecnología para el mejoramiento de la calidad de vida y, además, generar un imaginario tangible en el que el mercado se vislumbra desde una perspectiva humanista provoca en la sociedad una reconfiguración de las maneras de entenderse a sí misma.
En esta misma dinámica de construcción discursiva puede rastrearse entonces el primer indicio de mediación tanto comunicativa como pedagógica, ya que cuando el autor afirma que la convergencia de lo digital y lo educativo se desarrolla a partir de la creación de dispositivos interactivos, se está afirmando de manera tácita que estas formas de organización y puesta en circulación del saber están siendo definidas con intencionalidades precisas que parecen requerir, en el escenario virtual, un tratamiento diferente al de los contenidos académicos pensados para la educación presencial.

Esta afirmación se ve corroborada con apuestas que cambian profundamente las maneras de producción y circulación de propuestas de carácter académico, en las que la red se privilegia y maximiza discursos sobre el uso y optimización del tiempo, así como la interactividad y la autonomía. También garantiza la calidad del programa que se ofrece, sea este de formación de pregrado o de posgrado.

El nodo de la discusión está dado en la convergencia y sus bondades. La interactividad, como proceso comunicativo y pedagógico, supone un reto mayor, un desafío fundamentalmente marcado por la manera en la que se presenta esta propuesta pedagógica, a partir del fenómeno de la hibridación.

Proponer una producción de contenidos de carácter específico implica repensar y resignificar el escenario de producción, y no necesariamente el concepto en sí mismo.

La manera de desarrollar conceptos y contenidos tiene que ver directamente con la forma en la que estos son aprehendidos en primera instancia. La hibridación, por tanto, parte de una realidad en la que los escenarios de aprendizaje presencial, hacen de ella, en una primera interpretación, el producto de experiencias presenciales de enseñanza-aprendizaje trasladadas a escenarios virtuales, en los que se optimiza el aparato tecnológico y subyacen preguntas sobre las formas de desarrollar, resignificar, reapropiar y presentar estos contenidos.

Esta posibilidad de trasladar contenidos y estructuras pedagógicas del escenario presencial al escenario virtual genera un conjunto de disfunciones de orden didáctico en tanto que la construcción del mensaje, así como el modelo pedagógico adecuado para la circulación de este mensaje se piensa desde la técnica y no desde la mediación. 
Construir contenidos para el escenario virtual implica asumir niveles de complejidad que aún no tienen un cuerpo claramente definido. En este sentido, internet y las plataformas que a través de la red pueden configurarse, buscan validar un conjunto de escenarios interconectados, caracterizados por un alto nivel de lo simbólico que quieren generar apropiaciones en la enseñanza-aprendizaje.

Estos contenidos de alto nivel simbólico se configuran entonces con una lógica de la comunicación, en la que se pretende recuperar la acepción de comunicacióninformación, a la manera de un vaso comunicante. En esta metáfora la construcción discursiva de flujo rápido de información, y circulación de discursos que se anexan a un espacio definido, en ningún momento, aún en esta etapa, garantizan niveles de interpretación, argumentación o proposición en tanto que validación del concepto de aprendizaje, es decir, que la información circule a través de una plataforma, no garantiza de ninguna manera un proceso de enseñanzaaprendizaje.

Tras esta aproximación, un segundo aspecto para acercarse a su definición implica la construcción de una estructura monolítica en la que hay una serie de lineamientos de carácter instruccional, fundamentados en un conductismo clásico, que de una u otra manera, mediante mecanismos de control de tiempo-espacio, garantizan la realización, desde la perspectiva del creador-generador de contenidos, una serie de tareas.

Para contrarrestar esta didáctica, el escenario virtual ha generado un discurso fundamentado en los dispositivos de interacción e interconectividad que posibilitan las plataformas tales como grupos de trabajo colaborativo, chat, foro, trabajo individualizado de carácter asincrónico, evaluación abierta y programada desde la plataforma, tiempos y modalidades de evaluación por medio del diseño de pruebas de selección múltiple, selección única, actividades de relación lógica, el quiz, el ejercicio de refuerzo.

En esta validación, se establece entonces un tercer momento de comprensión de la mediación comunicativa y pedagógica de la hibridación y es la de estructurar contenidos que por medio de los dispositivos de interconectividad garanticen un aprendizaje real en tanto que apropiación del saber allí expuesto, situación que implica generar modelos pedagógicos que no responden a las características de la educación presencial, sino que deben confrontar esta virtualidad como un reto.
En este sentido, el modelo pedagógico puede pensarse como una espiral de constante configuración en la que, para privilegiar contenidos, lo espacio-temporal pasa a un segundo plano, en tanto que existen estructuras discursivas internas, que al mismo tiempo responden, por el mismo criterio de interconectividad, a una funcionalidad dada para el aprendizaje.

La hibridación, por tanto, cuenta con otro elemento para ser definida, la funcionalidad de los contenidos está dada por la pérdida parcial de lo espaciotemporal y, a la vez, apuesta por la posibilidad de la segmentación de los saberes, en una búsqueda desde lo homogenizante hacia la individualización de las búsquedas autónomas del saber.

El modelo pedagógico toma como base la conciencia del compromiso personal y, a la vez, la necesidad de configurar el concepto de red como respuesta a una responsabilidad colectiva y el valor del código como el elemento de interacción comunicativa en la circulación del mensaje.

Así, el modelo contribuye a desarrollar el criterio de comprensión del saber comunicado y de enmarcarlo en la noción de cooperación comunicativa, no como una extensión del primer concepto de comunicacióncomunitario, sino de comunicación como mediación de saberes. Es decir, con el parámetro de la cooperación, lo que se pretende es contribuir a la asimilación de los conceptos que ejercen cambios en el medio cognitivo y que por medio de la interconectividad serán discutidos con otros.

De esta manera, se crea la necesidad y el hábito de responder a una realidad siempre cambiante pero que, a la vez, genera otras experiencias de aprehender el mundo. El movimiento que presenta este modelo comunicativo es el espiral, en el que todos los elementos del proceso están en constante comunicación, respondiendo a la dinámica de él mismo.

El modelo comunicativo de espiral (Ballestas, 2000), nace de la interpretación de los elementos que han aportado los datos arrojados en el proceso investigativo, y está fundamentado desde el discurso de la transformación de la industria cultural a la industria educativa. Por ello, no es descabellado pensar que lo espacio-temporal de lo virtual, se configura para fortalecer la hibridación desde lo metafórico como herramienta pedagógica. 
El modelo retoma como punto de referencia de su propuesta, el espacio socio-cognitivo de la escuela, y desde este mismo punto desarticula el imaginario de una construcción para que se personifique y sea transformada la vida cotidiana.

Esta desarticulación propende por la configuración de un espacio cognitivo de persuasión en el que lo híbrido se centra en la co-construcción del individuo y genera un movimiento de descentralización de conceptos, que ya no pueden ser comprendidos desde las estructuras mentales del pasado, sino desde la concepción de un mundo mutuamente compartido, no solo físicamente sino también virtualmente.

El modelo presenta la apertura a otros puntos de vista y rescata la necesidad de la generación de nuevos espacios en los que contextualmente la comunicación se haga más rica. A su vez, propone la revisión constante de los enunciados y especialmente la construcción lingüística de cualquier propuesta pedagógica, tomando como base el arte de saber vivir, y su concreción desde la nueva concepción de educación y formación profesional para la transformación social.

Este discurso se valida cada vez más cuando comienzan a circular afirmaciones como:

La hibridación se acerca a tener un rol educativo destacado por la gratuidad y el bajo costo de acceso a los contenidos, el fácil aprendizaje y la poca capacitación para su uso, su estructura orientada al intercambio, su alta segmentación, la existencia de mecanismos de interacción que permiten la construcción de saberes colectivos en red (Rama, 2007).

La hibridación como un fenómeno comunicativo que se concibe abierto, obliga a preguntarse por su naturaleza como territorio. Desde esta inquietud es que la universidad como espacio creador gesta la idea, y para ello regresa a comprender cómo la educación es un punto de referencia culturalmente establecido por la sociedad. En esta misma línea, la educación como estructura aprehendida es una metáfora, ya que en ella se da el fenómeno de la metonimia que Lakoff y Johnson (1985) han planteado y que aborda el problema de la comprensión del nuevo concepto de educación y, a la vez, establece, en el modelo de comunicación, parámetros de virtualidad.

A partir de esta búsqueda de la comprensión, la metáfora de la industria educativa como territorio, comienza a reafirmarse, la hibridación también entra a formar parte de ese proceso nuevo, junto con elementos que enriquecen esta acción cognitiva. La teoría toma en cuenta criterios como la comunicación interpersonal virtual, la compresión mutua, desde la aplicación de comunidad virtual, la comprensión de la autonomía, la expresión estética y las apuestas en la mediación de los saberes.

Cuando el modelo de comunicación toma en cuenta estos aspectos, está visualizando la construcción de un discurso virtual, híbrido, no solo con la intencionalidad de propender por una industria educativa, sino a la vez por reafirmar que la metáfora es un elemento valioso para desarrollar procesos de aprendizaje, que si bien requieren de un tiempo y un espacio propios para madurar, pueden contribuir a aprehender de otra manera los saberes.

Así, bajo, la definición que Lakoff y Johnson (1985:242). Han denominado "le mythe expérientialiste": "La compréhension émerge de l'interaction, et d'une négotiation incessante avec l'environnement et les autres hommes"'. En su acceso al mundo la persona experimenta la realidad y la aprehende, ya no como una linealidad de apropiación de saberes, sino como un reflexionar y accionar co-construido Esta última observación reiterada en la hibridación: ya que la innovación permite deconstruir la industria cultural y resignificar la industria educativa.

A esta comprensión comunicativa, se va hilando dentro del texto el sentido del reconocimiento, no solo desde el otro, sino el reconocimiento del espacio de la educación.

Por su nueva dimensionalidad, este espacio posee limitantes de carácter tecnológico que hacen necesario optimizar el uso de los dispositivos que lo componen y en los que se hace referencia directa por ser generadores de tensión en términos de obstáculos reales pero que son superables. En este sentido, la metáfora no se queda en el limbo del enunciado de la virtualidad, sino que se convierte en característica de la hibridación que va contemplando elementos lingüísticos, que permiten el acceso a la esquematización mental de un modelo de espiral para la solución de los problemas propios del área del saber desde mediaciones comunicativas y pedagógicas híbridas.

Este momento comunicativo corresponde al entablar un diálogo directo y a crear otros niveles de conocimiento a partir del contacto que han establecido tanto los creadores de contenidos, como los usuarios de la

\footnotetext{
${ }^{1}$ Traducción: El mito experiencialista. La comprensión emerge de la interacción y de una negociación incesante con el entorno y con los otros.
} 
plataforma. La discusión es por tanto un espacio para reafirmar la realidad afrontada con la realidad que puede llegar a construirse. Es como si virtualmente el usuario hiciera una revisión de su saber y viera que en efecto las situaciones que ha abordado son producto del desarrollo de sus propias mediaciones.

Este aspecto detona en el usuario el replanteamiento constante de imaginarios, que se ve enfrentado a una situación totalmente nueva, donde por medio de su experiencia y con la colaboración de dispositivos tales como el trabajo colaborativo o la conformación y participación en la red o la comunidad virtual, puede llegar a hacer algo nuevo, se trata de una perspectiva nueva de configurar los saberes. En esta fase se adentran creadores de contenidos y usuarios al conocimiento; al comunicar el saber adquirido y a la vez a transformarlo.

Según Lakof y Johnson (1985: 127), "les métaphores nous permettent de comprendre un domaine d'expérience dans les termes d'un autre. Notre hypothèse est que la compréhension concene des domaines entiers d'expérience et non des concepts isóles" ${ }^{2}$, es decir, que solo se posibilita la comprensión de un enunciado mediante su contextualización, y las metáforas que conforman este enunciado adquieren la significación deseada, por tanto es factible validar tanto contenidos como la hibridación misma.

En este mismo escenario, el rehacer la discusión, como mediación comunicativa y pedagógica, implica contemplar la concreción de un dispositivo comunicativo que propenda por la hibridación, ya que en la aprehensión posible de situaciones cotidianas del aprendizaje, es factible rastrear los criterios propuestos en el modelo de comunicación de espiral y la definición de la industria educativa. Este dispositivo comunicativo permite abordar a través de lo virtual y la negociación, discernir constantemente la posibilidad de estar siendo homologados a una comunidad y pertenecer a una red.

La reconstitución del espacio privilegiado se logra siempre y cuando la metáfora de la hibridación como territorio sea desarrollada teniendo en cuenta otro dispositivo que propone la mediación, el de lo lúdico y lo estético, teniendo como fundamento la generación de conocimiento, descubriendo la importancia de las diferencias en la unidad, la diversidad cultural y todos aquellos conceptos que, generados desde lo metafórico se concretizan en situaciones de la vida cotidiana y en sí mismos van reapropiando la concepción del saber construido, del saber aplicado y del ejercicio profesional.

Así mismo, este espacio privilegiado no se desarrolla solamente en el plano de lo cognitivo, la participación virtual completa señala otros cambios. Lo cognitivo se traduce en gran parte en el actuar y como se ha planteado anteriormente, la sociedad contemporánea expresa su realidad en gran parte mediante un conjunto de lenguajes marcados por diversidad de estéticas y representaciones.

La aprehensión de la virtualidad y, por ende, de la hibridación como una totalidad permite la vivencia de experiencias que asumen el mundo con una óptica abierta. Si por medio de la experiencia el ser humano accede al mundo, es necesario reconfigurar desde lo espacio-temporal la concreción de las estructuras mentales que se desarrollan en el ambiente de la industria educativa.

Regresar sobre la fundamentación es una reflexión que todo el tiempo desarrolla la hibridación, ya que en ella como mediación se concreta la visión de la realidad y su modelo comunicativo, y pedagógico en el que se apela al dispositivo del aprendizaje en toda la dimensión que este alcance a desarrollar.

Por otra parte, subyacen en estas expresiones metafóricas nuevas significaciones que son definidas por Lakoff y Johnson como metáforas que proponen una significación diferente porque son el resultado de una creación, de la imaginación y están en el exterior del sistema conceptual ordinario, así como buscan incorporarse a un sistema virtual e híbrido.

Entonces, la utilización de estos instrumentos metafóricos también propone desde su interior la concepción de nuevas estructuras mentales para aprehender el mundo. Esta hibridación propone una nueva categorización de los valores, dejando como parte de la experiencia académica las estructuras mentales con las que se ha interpretado el mundo, y requiere ser visto desde una nueva valoración del mismo en tanto que realidad dinámica.

Un aspecto que es claro mediante de la expresión de una propuesta híbrida para los procesos de enseñanzaaprendizaje, es que se asuma como un reto para que la industria educativa como territorio sea desarrollada en el interior del modelo comunicativo como una co-cons-

2 Traducción. Las metáforas nos permiten comprender un dominio de experiencias en los términos del otro. Nuestra hipótesis es que la comprensión concierne a los dominios completos de la experiencia y no desde conceptos aislados. 
trucción de la realización pedagógica que está en constante retroalimentación.

\section{BIBLIOGRAFÍA}

Ballestas, Caro, Nancy. (2000). La métaphore comme outil de construction d'un modèle de communication pour une culture de la démocratie : comment communiquer des concepts abstraits ? Thèse de Maîtrise. Faculté de Sciences Économiques, Sociales et Politiques. Département de Communication. Louvain: Universitè Catholique de Louvain.

Lakoff, George; Johnson, Mark. (1985). Les métaphores dans la vie quotidiene. Paris: Ed. de Minuit, coll. Prepositions.

Rama, Claudio. (2007). La transformación de las industrias culturales en industrias educativas con la digitalización. Ponencia presentada en el seminario: El patrimonio cultural valenciano y su proyección iberoamericana. Valencia, España, 18-19 de abril. 